\title{
Intrauterine growth restriction coupled with hyperglycemia: effects on cardiac structure in adult rats
}

\author{
Kyungjoon Lim', Paul Lombardo², Michal Schneider-Kolsky² and M. Jane Black
}

BACKGROUND: Intrauterine growth restriction (IUGR) has been linked to heart disease in adulthood. Hence the IUGR heart is likely to be vulnerable to diabetic heart disease. The aim of this study was to examine the effect of induction of type 1 diabetes on myocardial collagen deposition and cardiac function in adult rats with a history of IUGR, after controlling blood glucose levels.

METHODS: IUGR was induced by protein restriction in the pregnant female rat. When the offspring were 24 wk of age, diabetes was induced in male IUGR and non-IUGR rats by means of streptozotocin; insulin injections were used to maintain blood glucose levels at a mild ( $7-10 \mathrm{mmol} / / ; n=8$ per group) or moderate level (10-15 mmol//; $n=8$ per group). Echocardiography and cardiac morphology analyses were carried out when the rats were 32 wk of age.

RESULTS: IUGR offspring exhibited cardiac hypertrophyat 32 wk, including a thicker posterior wall and increased interstitial fibrosis in the left ventricle. Hyperglycemia led to an increase in heart size and myocardial fibrosis. The response to hyperglycemia was not different between IUGR and non-IUGR rats; however, cardiac fibrosis was greatest when diabetes was present along with a history of IUGR. In general, maintaining blood glucose levels at a mildly hyperglycemic level attenuated the adverse effects of hyperglycemia but did not reverse the fibrosis.

CONCLUSION: Exacerbated fibrosis in hyperglycemic hearts of IUGR offspring may lead to long-term cardiac dysfunction.

l:

$\mathrm{n}$ recent years there has been an escalation in the incidence of diabetes mellitus (type 1 and type 2 ) in both developing $(1,2)$ and developed countries $(3,4)$; this escalation is associated with increased mortality $(5,6)$ and morbidity $(7,8)$ worldwide. Diabetes leads to pathological remodeling of the myocardium, resulting in loss of cardiomyocytes (9) and subsequent deposition of fibrillar collagen (fibrosis) (10); heart failure can subsequently ensue $(11,12)$.

Of note, recent epidemiological studies demonstrate that the foundations for adult disease may be established in early life, with intrauterine growth restriction (IUGR) strongly linked to the development of ischemic heart disease in adulthood $(13,14)$. In animal models, there is clear evidence of cardiac programming in IUGR offspring as a result of prenatal hypoxia (15). In the rodent maternal protein restriction model, previous studies have shown increased collagen deposition in the hearts of IUGR offspring by early adulthood (24 wk of age) (16) and overt cardiac dysfunction in these animals by $40 \mathrm{wk}$ of age (17). Therefore in this study we proposed that IUGR acts as an initial insult to the developing heart, rendering it more vulnerable to secondary postnatal insults. We hypothesized that there will be synergistic deleterious effects on cardiac function when a secondary insult such as diabetes is added to the initial developmental insult of IUGR; we further hypothesized that these adverse effects would be attenuated by maintaining blood glucose levels at a mildly hyperglycemic level. The aim of this study, therefore, was to examine the effects of induction of type 1 diabetes on cardiac structure and function in adult rats with a history of IUGR when blood glucose was maintained at either a mildly or moderately increased level. For this purpose we have conducted echocardiographic tests of heart function and quantitative analyses of cardiac fibrosis in rats that were growth restricted in utero (as a result of maternal protein restriction) and then challenged later in life with the induction of type 1 diabetes as the secondary insult.

\section{RESULTS}

\section{Growth Trajectories}

Body weight. The offspring born to pregnant rats that were fed a low-protein diet (LPD) to induce IUGR are referred to here as "LPD offspring." Offspring born to rats that had a normal-protein diet (NPD) are referred to as "NPD offspring." At $3 \mathrm{~d}$ of age, the LPD offspring had body weights (BWTs) of $4.44 \pm 0.13 \mathrm{~g}$ which were $24 \%$ lower than the weights of NPD offspring $(5.74 \pm 0.16 \mathrm{~g})(P<0.001)$. At $32 \mathrm{wk}$ of age, the BWTs of the LPD offspring remained significantly lower than those of the NPD offspring (10\% lower, $P$ value relating to maternal diet $\left.\left(P_{\mathrm{D}}\right)<0.0001\right)$ (Figure 1a). When type 1 diabetes was induced, the increasing levels of blood glucose reduced ( $P$ value relating to hyperglycemic treatment $\left(P_{\mathrm{T}}\right)<0.0001$ ) the BWTs in LPD offspring as well as in NPD offspring. The maintenance of blood glucose levels at a mildly hyperglycemic level attenuated the effects of hyperglycemia on BWT in NPD offspring (Figure 1a); however, the LPD offspring with mild hyperglycemia remained significantly smaller than the LPD control offspring. 

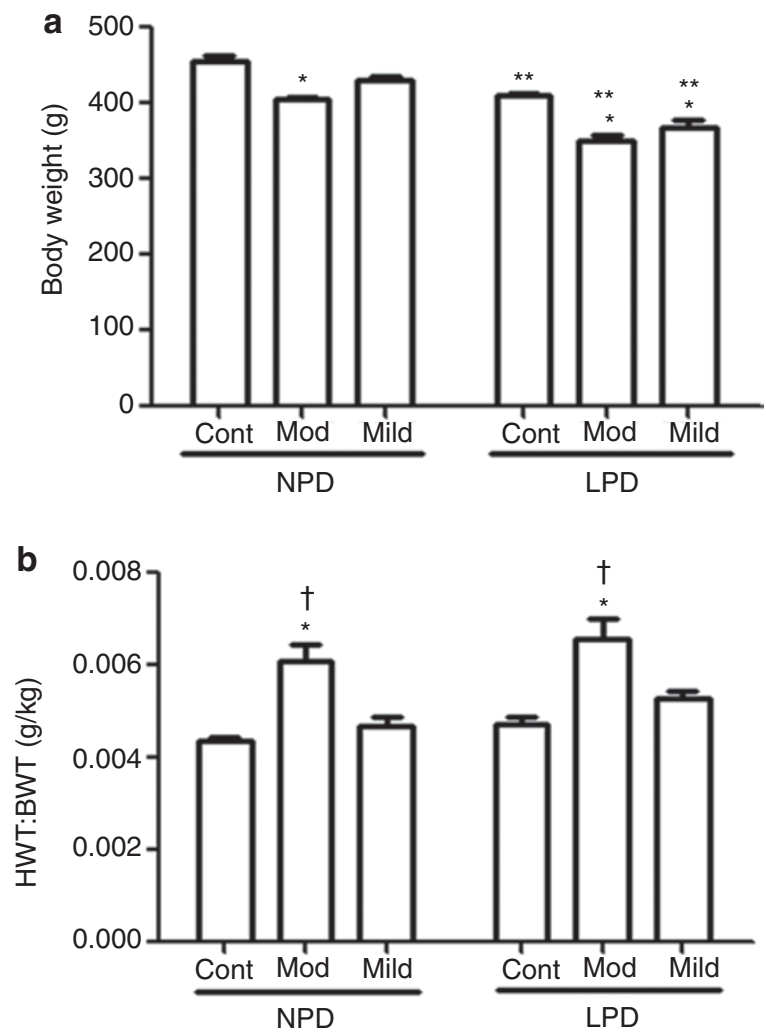

Figure 1. Body weight and relative heart weight. (a) Body weight and (b) heart weight:body weight ratio (HWT:BWT) at 32 wk of age in NPD and LPD control (Cont) offspring and those exposed to mild or moderate (Mod) hyperglycemia from 24 to $32 \mathrm{wk}$ of age ( $n=8$ per group). LPD offspring exhibited a lower body weight $\left(P_{\mathrm{D}}<0.0001\right)$ at $32 \mathrm{wk}$ of age. Hyperglycemia led to a marked decrease in body weight $\left(P_{T}<0.0001\right)$ as shown in (a). HWT:BWT was significantly higher in LPD offspring $\left(P_{D}\right.$ $=0.05)$. The induction of hyperglycemia further increased the HWT:BWT ratio $\left(P_{T}<0.0001\right)$. Data were analyzed using a two-way ANOVA, the factors being maternal diet $\left(P_{\mathrm{D}^{\prime}}\right.$ LPD or NPD), hyperglycemic treatment $\left(P_{\mathrm{T}^{\prime}}\right.$ control, mild hyperglycemia, or moderate hyperglycemia), and their interaction $\left(P_{\mathrm{DXT}}\right)$; post hoc analyses were performed to compare data within the diet groups: ${ }^{*} P<0.05$ vs. control, within the diet groups: ${ }^{* *} P<0.05$ for LPD group vs. respective NPD group, and ${ }^{\dagger} P<0.05$ for mild vs. moderate hyperglycemia. LPD, low-protein diet; NPD, normal-protein diet.

Heart weight. There was no significant difference in absolute heart weight in LPD offspring as compared with NPD offspring $(1.95 \pm 0.07$ and $1.98 \pm 0.04$, respectively). However, when heart weights were adjusted to BWTs, LPD offspring exhibited significantly higher $\left(P_{\mathrm{D}}=0.05\right)$ relative heart weights as compared with NPD offspring at $32 \mathrm{wk}$ of age (Figure $1 \mathrm{~b}$ ). The induction of hyperglycemia led to a marked increase $\left(P_{\mathrm{T}}=0.0002\right)$ in absolute heart weight in both LPD and NPD offspring. Furthermore, relative heart weight was also higher in all groups with hyperglycemia $\left(P_{\mathrm{T}}<0.0001\right)$, with moderately hyperglycemic LPD offspring exhibiting the highest heart weight relative to BWT (Figure 1b).

\section{Blood Pressure}

Blood pressure remained constant throughout the measurement period, from 24 to $32 \mathrm{wk}$ of age, in both LPD and NPD offspring (Figure 2a). There was no significant effect of hyperglycemia on blood pressure over the experimental period (Figure 2a).
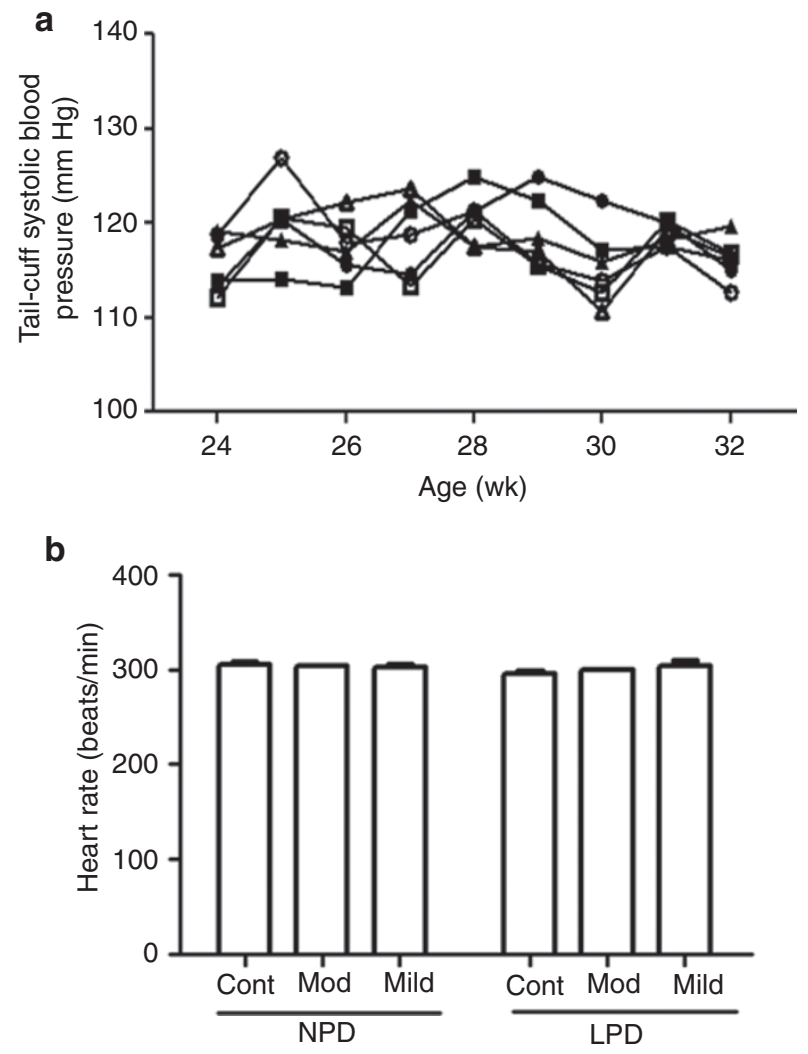

Figure 2. Blood pressure and heart rate. (a) Tail-cuff systolic blood pressure from 24 to 32 wk of age and (b) heart rate in NPD and LPD control (Cont) offspring and those exposed to mild or moderate (Mod) hyperglycemia ( $n=8$ per group). (NPD: control (filled circles), mild (filled squares), moderate (filled triangles); and LPD: control (open circles), mild (open squares), moderate (open triangles)). Data were analyzed using a repeated two-way ANOVA for blood pressure over time, and a two-way ANOVA for heart rate with the factors being maternal diet $\left(P_{\mathrm{D}^{\prime}}\right.$ LPD or NPD), hyperglycemic treatment ( $P_{\mathrm{T}}$; control, mild, or moderate), and their interaction $\left(P_{\mathrm{D} \times \mathrm{T}}\right)$. LPD, low-protein diet; NPD, normal-protein diet.

\section{Echocardiographic Analyses}

Heart rate. Heart rate was not affected by maternal protein restriction or the induction of hyperglycemia in either LPD or NPD offspring at $32 \mathrm{wk}$ of age (Figure $2 \mathbf{b}$ ).

End-diastolic internal diameter of the left ventricle. Maternal protein restriction during pregnancy and lactation led to a significant reduction $\left(P_{\mathrm{D}}<0.0001\right)$ in the end-diastolic internal diameter of the left ventricle (LVIDd) of LPD offspring as compared with control NPD offspring (Figure 3a). There was a marked increase in the LVIDd in response to the induction of hyperglycemia $\left(P_{\mathrm{T}}=0.0008\right)$. However, the response to hyperglycemia was not significantly different between LPD and NPD offspring $\left(P_{\mathrm{D} \times \mathrm{T}}=0.2\right)$. Post hoc analyses demonstrated a significant increase $(P<0.05)$ in the LVIDd in LPD offspring with moderate hyperglycemia as compared with LPD control offspring; however, the LVIDd was normalized when blood glucose levels were reduced to mildly hyperglycemic levels.

When the LVIDd was adjusted for BWT, the relative value of LVIDd was not different between LPD and NPD offspring (Figure 3b); however, the induction of hyperglycemia led to a marked increase $\left(P_{\mathrm{T}}<0.0001\right)$ in the ratio of LVIDd:BWT. 

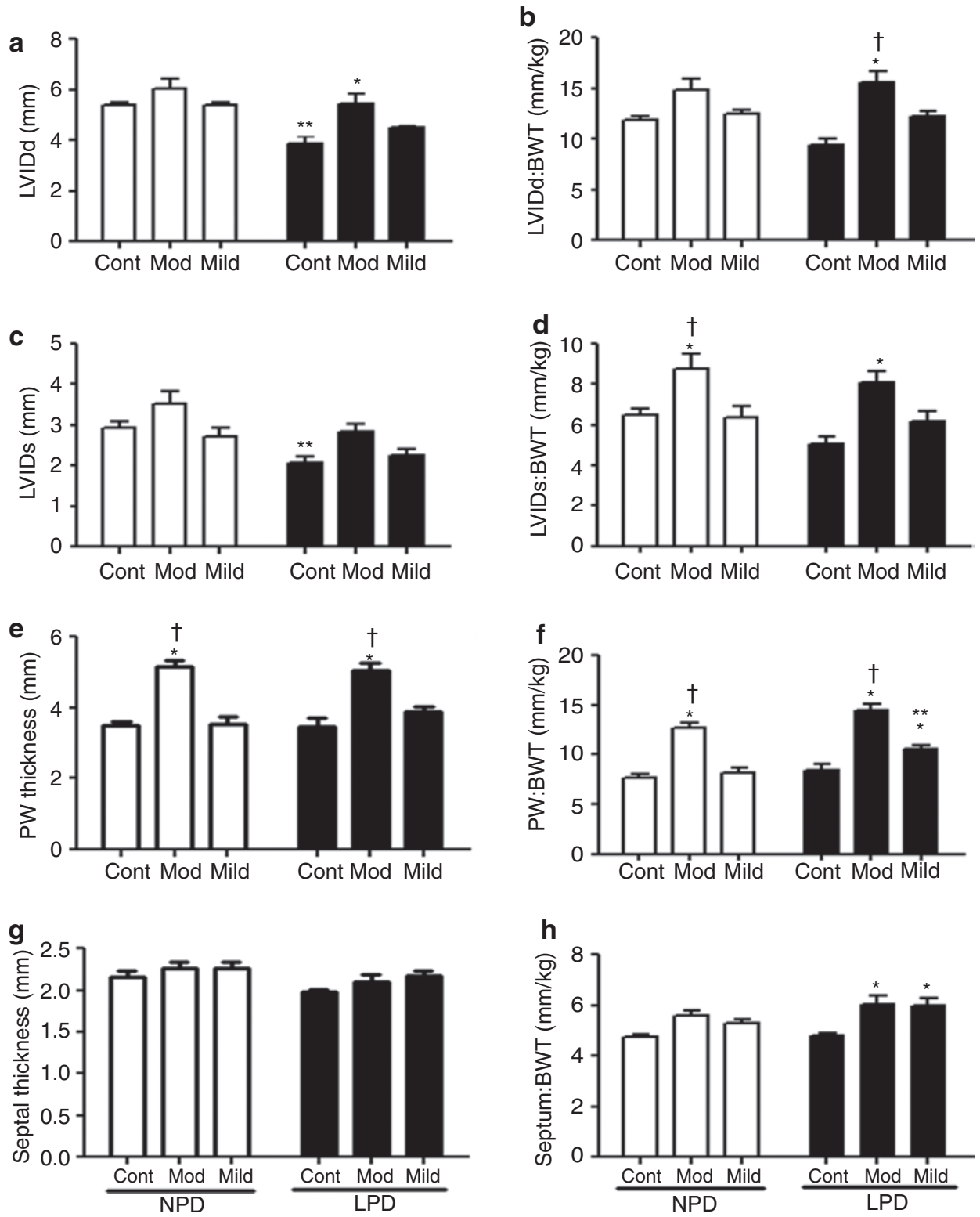

Figure 3. Echocardiographic determination of cardiac internal structure at 32 wk of age in NPD (white bars) and LPD (black bars) control (Cont) offspring and those exposed to mild or moderate (Mod) hyperglycemia from 24 to $32 \mathrm{wk}$ of age ( $n=8$ per group). End-diastolic internal diameter of the left ventricle (LVIDd) was reduced in LPD offspring $\left(P_{\mathrm{D}}<0.0001\right)$. (a) Hyperglycemia significantly increased $\left(P_{\mathrm{T}}=0.0008\right)$ LVIDd. (b) LVIDd relative to body weight (LVIDd:BWT) was significantly increased $\left(P_{\mathrm{T}}<0.0001\right)$ in rats with hyperglycemia. (c) End-systolic internal diameter of the left ventricle (LVIDs) was reduced $\left(P_{\mathrm{D}}=0.0002\right)$ in LPD offspring as compared with NPD offspring. Hyperglycemia had the effect of increasing $\left(P_{\mathrm{T}}=0.001\right) \mathrm{LVIDs}$. (d) LVIDs relative to body weight (LVIDs:BWT) was significantly increased $\left(P_{\mathrm{T}}<0.0001\right)$ in hyperglycemic offspring. (e) Posterior wall (PW) thickness was significantly increased in hyperglycemic offspring. (f) PW thickness relative to BWT (PW:BWT) was significantly increased $\left(P_{\mathrm{D}}=0.0001\right)$ in LPD offspring, and hyperglycemia also had the effect of increasing $\left(P_{\mathrm{T}}<0.0001\right)$ PW:BWT. (g) Septal thickness was significantly reduced $\left(P_{\mathrm{D}}=0.01\right)$ in LPD offspring. (h) Septal thickness relative to BWT (septum:BWT) was significantly increased $\left(P_{\mathrm{D}}=0.05\right)$ in LPD offspring, and hyperglycemia also had the effect of increasing $\left(P_{\mathrm{T}}<0.0001\right)$ septum:BWT. The data were analyzed using a two-way ANOVA, the factors considered being: maternal diet $\left(P_{\mathrm{D}} ; \mathrm{LPD}\right.$ or NPD), hyperglycemic treatment $\left(P_{\mathrm{T}} ;\right.$ mild or moderate), and their interaction $\left(P_{\mathrm{DXT}}\right)$. Post hoc analyses were performed to compare data within the diet groups: ${ }^{*} P<0.05$ vs. control, within the diet groups: ${ }^{* *} P<0.05$ for LPD group vs. respective NPD group, and ${ }^{\dagger} P<0.05$ for mild vs. moderate hyperglycemia. LPD, low-protein diet; NPD, normal-protein diet.

When blood glucose levels were maintained at a mildly hyperglycemic level in LPD and NPD offspring, the LVIDd:BWT ratio was not significantly different as compared with their respective control groups.

End-systolic internal diameter of the left ventricle. As seen in the LVIDd, there was a significant decrease $\left(P_{\mathrm{D}}=0.0002\right)$ in end-systolic left-ventricular dimension in LPD offspring as compared with NPD offspring (Figure 3c). Increasing levels of blood glucose led to marked increases $\left(P_{\mathrm{T}}=0.001\right)$ in endsystolic internal diameter of the left ventricle (LVIDs) in both LPD and NPD offspring.

When the LVIDs was adjusted for BWT, there was no significant difference between LPD and NPD offspring $\left(P_{\mathrm{D}}=0.08\right)$ 
as regards the LVIDs:BWT ratio (Figure 3d). Hyperglycemia led to a marked increase $\left(P_{\mathrm{T}}<0.0001\right)$ in the LVIDs:BWT ratio; when blood glucose levels were reduced in the mildly hyperglycemic rats, there was no significant difference in the LVIDs:BWT ratio relative to their respective controls.

Posterior wall thickness. Maternal protein restriction during pregnancy and lactation did not affect posterior wall (PW) thickness in the resulting LPD offspring as compared with NPD offspring (Figure 3e). Induction of hyperglycemia led to a marked increase $\left(P_{\mathrm{T}}<0.0001\right)$ in PW thickness in both LPD and NPD offspring. When adjusted for BWT, there was a significant increase $\left(P_{\mathrm{D}}=0.0001\right)$ in relative PW thickness in LPD offspring (Figure 3f). Hyperglycemia lead to a marked increase $\left(P_{\mathrm{T}}<0.0001\right)$ in the LVIDs:BWT ratio; however, when blood glucose levels were reduced in the mildly hyperglycemic rats, there was no significant difference in the LVIDs:BWT ratio relative to their respective controls. However, by lowering blood glucose levels (in mildly hyperglycemic groups), the absolute PW thickness in both LPD and NPD offspring, and the relative PW thickness in NPD offspring were restored to the levels of the respective control groups. However, the relative PW thickness in LPD offspring remained elevated even after blood glucose levels were reduced to a mildly hyperglycemic level.

Septal thickness. Overall, LPD offspring exhibited a significantly reduced $\left(P_{\mathrm{D}}=0.01\right)$ septal thickness as compared with NPD offspring (Figure 3g). The induction of hyperglycemia did not affect the septal thickness in LPD offspring relative to NPD offspring. When adjusted for BWT, there was a significantly higher $\left(P_{\mathrm{D}}=0.05\right)$ ratio of septum:BWT in LPD offspring as compared with NPD offspring (Figure $3 \mathrm{~h}$ ). Increases in the levels of blood glucose also significantly increased $\left(P_{\mathrm{T}}\right.$ $<0.0001)$ the septum:BWT ratio. Unlike other parameters, relative septal thickness in LPD offspring was not restored to control levels by maintaining blood glucose levels at a mildly hyperglycemic level.

Fractional shortening. Fractional shortening (FS) was not different in LPD offspring as compared with NPD offspring (Figure 4). Hyperglycemia did not alter FS in either LPD or NPD offspring.

\section{Cardiac Fibrosis}

Interstitial/reparative fibrosis. There was a significant increase $\left(P_{\mathrm{D}}<0.0001\right)$ in the levels of interstitial fibrosis in the left ventricle plus septum $(\mathrm{LV}+\mathrm{S})$ of LPD offspring as compared with those of NPD offspring (Figure 5). Both LPD and NPD offspring responded in a similar manner to hyperglycemia, with marked increases $\left(P_{\mathrm{T}}<0.0001\right)$ in interstitial fibrosis in the $\mathrm{LV}+\mathrm{S}$. Even when blood glucose levels were maintained at a mildly hyperglycemic level, the extent of interstitial fibrosis was not normalized in either LPD or NPD offspring.

LPD offspring showed no difference relative to NPD offspring as regards the levels of interstitial fibrosis in the right ventricle (RV), with or without hyperglycemia. There was no

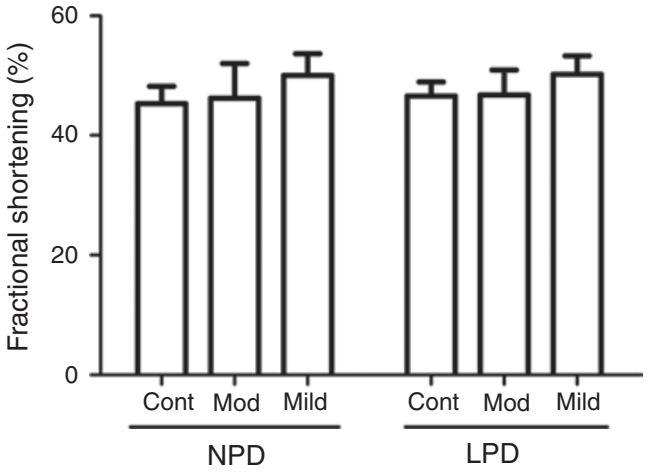

Figure 4. Left ventricular fractional shortening in NPD and LPD control (Cont) offspring and those exposed to mild or moderate (Mod) hyperglycemia from 24 to 32 wk of age ( $n=8$ per group). LPD, low-protein diet; NPD, normal-protein diet.

evidence of reparative fibrosis in either the $\mathrm{LV}+\mathrm{S}$ or $\mathrm{RV}$ of $\mathrm{LPD}$ and NPD offspring at $32 \mathrm{wk}$ of age.

Perivascular fibrosis. There was no difference in the adventitia:lumen ratio in LPD control offspring as compared with NPD control offspring at $32 \mathrm{wk}$ of age. The induction of hyperglycemia did not affect the adventitia:lumen ratio in either LPD or NPD animals.

Maternal LPD and hyperglycemia did not affect the levels of perivascular fibrosis in the $\mathrm{LV}+\mathrm{S}$ or RV of any of the offspring.

Media-to-lumen ratio of intramyocardial arteries. There was no significant difflerence between LPD and NPD offspring in the media:lumen ratio of intramyocardial arteries in either the $\mathrm{LV}+\mathrm{S}$ or RV.

\section{DISCUSSION}

The findings of this study demonstrate the induction of left-ventricular hypertrophy in adult rats with a history of IUGR. This was accompanied by a thicker posterior wall relative to body weight and increased interstitial fibrosis. As expected, there were indications of altered cardiac function (enlarged end-diastolic and end-systolic dimensions) when type 1 diabetes was induced in the animals; in general, the IUGR and control rats did not differ in respect of their response to hyperglycemia. Maintaining blood glucose levels at a mildly hyperglycemic level markedly attenuated the adverse effects of type 1 diabetes; however, the increase in collagen deposition was not reversed.

\section{Altered Cardiac Growth in Offspring With IUGR}

There was clear evidence of altered cardiac growth in LPD (IUGR) offspring as compared with controls. The observed increase in relative heart weight in the IUGR rats is indicative of left-ventricular hypertrophy in these animals which is supported by the observed increase in relative PW thickness in the left ventricle. Left-ventricular hypertrophy has been described in other experimental models of IUGR as well (18$20)$, but this is often associated with an elevation in arterial 

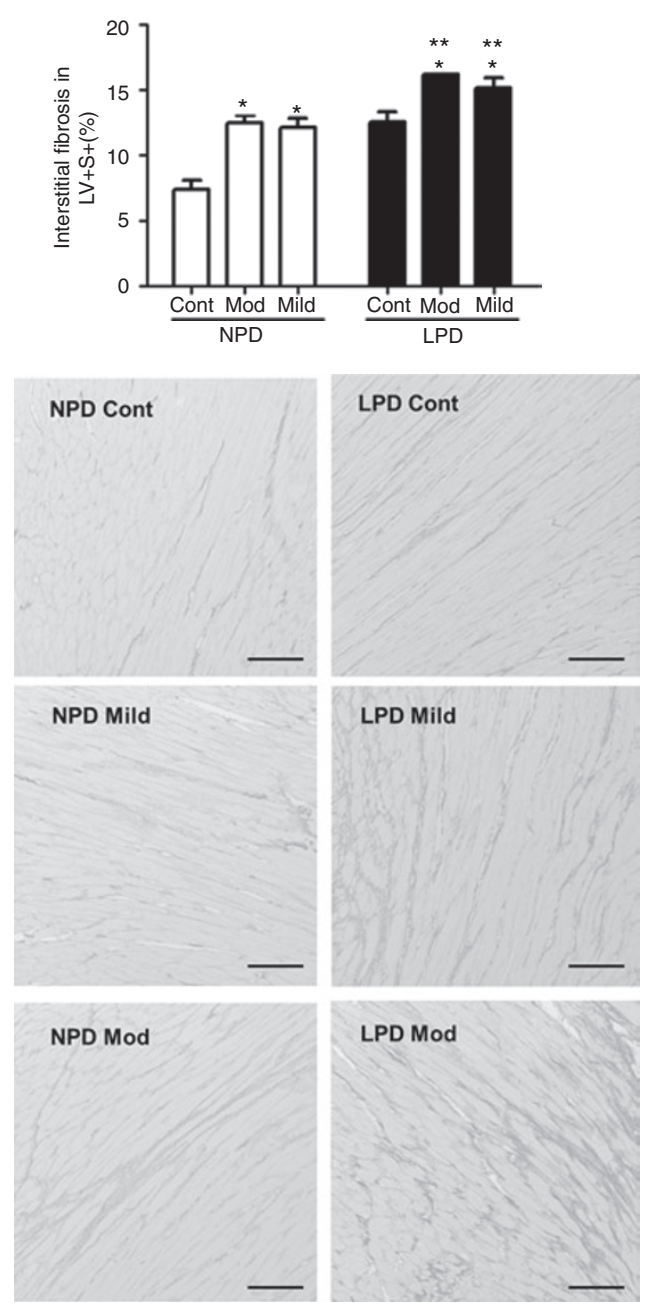

LPD Mod

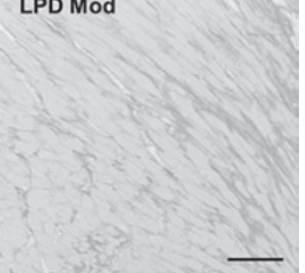

Figure 5. Analysis of picrosirius-red stained sections of the left ventricle. The top panel represents the percentage of interstitial fibrosis of the $\mathrm{LV}+\mathrm{S}$ (the open bars represent NPD offspring with control (Cont), mild, and moderate (Mod) hyperglycemic groups; the filled bars represent LPD offspring with control (Cont), mild, and moderate (Mod) hyperglycemic groups). LPD offspring exhibited significantly higher levels $\left(P_{D}<0.0001\right)$ of interstitial fibrosis, and hyperglycemia also had the effect of increasing $\left(P_{\mathrm{T}}<0.0001\right)$ the level of interstitial fibrosis. The data were analyzed using a two-way ANOVA, the factors considered being maternal diet $\left(P_{\mathrm{D}^{\prime}} \mathrm{LPD}\right.$ or NPD), hyperglycemic treatment $\left(P_{\mathrm{T}}\right.$ control, mild, or moderate), and their interaction $\left(P_{\mathrm{DXT}}\right)$. Post hoc analyses within the diet groups: ${ }^{*} P<0.05$ vs. control; ${ }^{* *} P<0.05$ for LPD group vs. respective NPD group. The bottom panels represent picrosirius red-stained sections ( $\times 200$ magnification; bar $=50 \mu \mathrm{m}$ ) showing interstitial collagen fibers (stained red) in the LV+S of hearts from NPD control (Cont), mild, and moderate (Mod) hyperglycemic groups, and from LPD control (Cont), mild, and moderate (Mod) hyperglycemic groups. LPD, low-protein diet; LV+S, left ventricle plus septum; NPD, normal-protein diet.

blood pressure $(19,20)$. Of note, left-ventricular hypertrophy in the IUGR offspring in our study appears to have developed independently of a change in blood pressure; when tail-cuff blood pressure was measured from 24 to $32 \mathrm{wk}$ of age there was no difference in blood pressure between the LPD and NPD offspring. An absence of blood pressure differences in adulthood between LPD and NPD offspring is a consistent finding in our laboratory, where we have used this model of maternal protein restriction during pregnancy and lactation, including studies in which conscious blood pressure was measured using an indwelling catheter $(21,22)$. In addition, similar findings of no effects on long-term blood pressure in IUGR offspring have been reported by other laboratories (23-26).

The relative increase in heart size in the LPD offspring is probably the result of increased collagen deposition within the myocardium; there was a marked increase in interstitial fibrosis within the myocardium of the IUGR offspring. These findings are in accordance with previous findings in our laboratory; earlier, we observed a 15\% increase in interstitial fibrosis in the hearts of LPD offspring at $24 \mathrm{wk}$ of age (16). A similarly significant increase in interstitial fibrosis has been described in adolescent guinea pigs that were growth restricted in utero because of chronic placental insufficiency (27).

Alternatively, the increase in relative heart weight may be attributable to an increase in cardiac muscle mass as a result of cardiomyocyte hypertrophy; cardiomyocytes generally do not proliferate in the adult heart. In this context, the administration of insulin may have mediated some of the effects. Indeed, insulin treatment has been shown to induce hypertrophy in cultured cardiomyocytes (28), and chronic hyperinsulinemia (29) has been shown to lead to cardiac hypertrophy in rats. Whether cardiomyocyte growth was affected and/or mediated by insulin in our study requires further investigation.

Although cardiac fibrosis is a well known cause of altered contractility of the myocardium $(30,31)$, left-ventricular contractility appears to be adequately maintained at this stage, as seen from the finding that FS of the cardiac muscle did not differ between the LPD and NPD offspring. It appears likely that the left-ventricular hypertrophy observed in the IUGR offspring leads to encroachment on the left ventricle chamber size during systole, as judged from the significant decrease in end-systolic dimensions in the hearts of IUGR animals.

Induction of Type 1 Diabetes Leads to Impaired Cardiac Function Although diabetes mellitus is defined as a metabolic disorder, the major direct cause of morbidity and mortality in diabetes is from cardiovascular disease $(32,33)$. It has been shown that patients with diabetes exhibit changes in cardiac structure such as ventricular and myocyte hypertrophy $(34,35)$, increased deposition of collagen $(11,36)$, and advanced glycation end products (37). Patients with diabetes have an increased susceptibility to developing heart failure after myocardial infarction and, once established, the outcome is worse than in patients without diabetes (38). In accordance with the literature, in this study we found that the induction of hyperglycemia led to leftventricular hypertrophy and altered cardiac structure. When assessed echocardiographically, there was a significant increase in relative end-diastolic diameter of the left ventricle, suggesting impairment of diastolic function (39). In addition, there was a significant increase in relative end-systolic dimensions, which may reflect the induction of left-ventricular hypertrophy consequent to hyperglycemia. Of note, the induction of hyperglycemia led to a marked increase in interstitial fibrosis in both NPD and LPD offspring. 
Induction of Type 1 Diabetes in IUGR Offspring

Epidemiological studies closely link IUGR with long-term cardiovascular disease (40-42). Therefore in this study we proposed that IUGR would act as an initial insult on the growth of the heart, rendering it vulnerable to a subsequent secondary insult. The secondary cardiovascular insult initiated in this study was the induction of type 1 diabetes, resulting in hyperglycemia. Of note, we found that the response to hyperglycemia was not different between the LPD and NPD offspring; there was no significant relationship between maternal diet and hyperglycemia with respect to any of the experimental parameters examined. There was therefore no evidence of an exaggerated pathological response to hyperglycemia in the hearts of adult rats that had been subjected to IUGR by maternal protein restriction.

\section{Maintaining Blood Glucose Levels at a Mildly Hyperglycemic Level Attenuates Left-Ventricular Hypertrophy in Control Rats but Does Not Attenuate Cardiac Fibrosis}

When blood glucose levels were maintained at a mildly hyperglycemic level in the diabetes-induced NPD offspring, the extent of cardiac hypertrophy (including PW thickness) was markedly attenuated and not different from the values in controls with no diabetes. An increase in relative LVIDd (indicative of diastolic dysfunction (39)) was seen in the NPD offspring maintained at moderately hyperglycemic levels, whereas this was not observed in the NPD group in which blood glucose levels were maintained at a mildly hyperglycemic level. Of note, however, these beneficial effects of maintaining blood glucose levels at a mildly hyperglycemic level were not as pronounced in the LPD offspring with respect to cardiac hypertrophy; the relative posterior left-ventricular wall thickness and the relative septum thickness, although attenuated, remained significantly higher than in the respective controls. This may, in part, reflect the presence of high levels of fibrosis in the hearts of the LPD offspring. A finding that is of particular concern in this regard was that the increase in interstitial fibrosis as a result of the induction of diabetes was not attenuated with improved glycemic control in either the NPD or the LPD offspring. The levels of interstitial fibrosis were already elevated in LPD offspring and, with the induction of hyperglycemia, these levels were further exacerbated in both the mildly and the moderately glycemic LPD groups as compared with the respective NPD groups, as assessed using post hoc analyses. Given that cardiac fibrosis is a major cause of cardiac dysfunction, our results suggest that, in adulthood, offspring with a history of IUGR may be at heightened risk of cardiac dysfunction in the presence of hyperglycemia as compared with those without such a history, even when blood glucose levels are maintained at a mildly hyperglycemic level.

An alternative explanation for the persistently high levels of fibrosis in the hearts of the animals maintained at mildly or moderately hyperglycemic levels may relate to the administration of insulin. Further investigation is required to determine the contribution of insulin to the increased levels of myocardial fibrosis that we found in the animals in our study.
In conclusion, we have shown cardiac hypertrophy accompanied by increased myocardial collagen deposition in adult rats that were growth restricted in utero. The induction of hyperglycemia also led to an increase in heart size and myocardial fibrosis. The response to hyperglycemia in adulthood was not different between the animals with a history of IUGR and those without. However, the levels of cardiac fibrosis were greatest when adult-onset diabetes was combined with a history of IUGR. Although maintaining blood glucose levels at a mildly hyperglycemic level in rats with diabetes did, in general, attenuate the abnormal effects on cardiac growth and normalize end-diastolic and end-systolic left-ventricular diameters, the increases in myocardial fibrosis were not attenuated. Therefore, although the function of the cardiac muscle (as assessed by FS) was not compromised in any of the offspring studied, it is likely that the exacerbated myocardial fibrosis observed in the hyperglycemic offspring with a history of IUGR could lead to overt cardiac muscle dysfunction in later life, even if glucose levels are well maintained.

\section{METHODS}

\section{Animals and Diet Treatment}

Female Wistar Kyoto breeder rats were obtained from the Australian Resource Centre (Perth, Australia). The female rats were divided into two groups and fed either a NPD $(n=8 ; 20 \%$ casein) or a LPD ( $n=8 ; 8.7 \%$ casein) for 2 wk before mating (to familiarize the dams with the diets), during pregnancy, and for $2 \mathrm{wk}$ after delivery of the litter (because cardiomyocytes continue to proliferate in the first $2 \mathrm{wk}$ after birth in rats (43)). After this, all the dams were fed normal laboratory chow. The semipurified diets were ones that were commercially available (Glen Forrest Stockfeeders, Perth, Australia). The nutrient contents of the NPD and LPD semipurified diets were equivalent except for starch, which was varied to ensure that the diets were close to isocaloric (16). The breeder rats were housed individually and maintained at an ambient temperature of $21^{\circ} \mathrm{C}$, with a 12 -h day/night cycle. Food and water was administered ad libitum. To prevent stress to the dams, the pups were not handled until $3 \mathrm{~d}$ after birth. At postnatal day 3 , all the litters were reduced to 8 pups per litter. Litter sizes ranged from 8 to 12 pups, and there was no difference in litter size between groups.

At weaning, NPD and LPD offspring were separated into three subgroups, each with eight male offspring; only one male weanling per litter per group was used. At $24 \mathrm{wk}$ of age, diabetes was induced by a single intraperitoneal injection of streptozotocin (STZ; Sigma-Aldrich, St Louis, MO, $50 \mathrm{mg} / \mathrm{kg}$ in $0.1 \mathrm{~mol} / \mathrm{l}$ citrate buffer solution) in two of the experimental groups. The rest of the animals were injected with vehicle (control group). Three days after STZ injection, blood glucose levels were measured to ensure that the rats had developed diabetes (blood glucose level $>15 \mathrm{mmol} / \mathrm{l}$ ). Before injecting STZ, there was no difference in blood glucose levels between the LPD and NPD offspring $(4.03 \pm 0.07$ and $4.16 \pm 0.04 \mathrm{mmol} / \mathrm{l}$, respectively). From day 3 after STZ injection to $32 \mathrm{wk}$ of age, blood glucose levels were maintained at either a mildly hyperglycemic $(7-10 \mathrm{mmol} / \mathrm{l})$ level (mildly hyperglycemic group) or at a moderately hyperglycemic $(10-15 \mathrm{mmol} / \mathrm{l})$ level (moderately hyperglycemic group). Blood glucose levels were measured daily in all the animals at 4:00 PM from a drop of blood drawn from the tail artery, and short- and long-acting doses of insulin (Actrapid and/or Protaphane; Novo Nordisk, Baulkham Hills, Australia) were injected subcutaneously (1 IU of short-acting insulin Actrapid, followed by 1-3 IU of long-acting insulin Protaphane $2 \mathrm{~h}$ after the initial Actrapid injection) to maintain blood glucose levels within the predetermined range. There was no difference in the amounts of insulin administered to the LPD and NPD offspring to maintain the targeted glycemic levels. The experiments were approved by the 
Monash University Biochemistry, Anatomy, and Microbiology Animal Ethics Committee. The treatment and care of the animals conformed to the Australian Code of Practice for the care and use of animals for scientific purposes (Office of Laboratory Animal Welfare: A5140-01). All experiments were in conformance with the Guide for the Care and Use of Laboratory Animals published by the US National Institutes of Health (NIH publication no. 85-23, revised 1996).

\section{Tail-Cuff Systolic Blood Pressure Measurement}

Systolic blood pressure was measured in pre-warmed restrained rats (NPD and LPD offspring) from 24 to $32 \mathrm{wk}$ of age using tail-cuff sphygmomanometry. The rats were familiarized with the procedure starting $1 \mathrm{wk}$ before the actual measurement so as to reduce stress effects of tail-cuff measurement. The blood pressure measurements were performed in triplicate at 9:00 AM, twice a week.

\section{Echocardiographic Analyses}

Echocardiographic analyses of cardiac dimensions and function were performed in the experimental animals at $32 \mathrm{wk}$ of age, using a Philips HDI5000 SonoCT ultrasound machine (Philips Healthcare, North Ryde, Australia) with a $12-5 \mathrm{MHz}$ linear array transducer. A "pediatric-neonatal renal" setting was used with a focal depth of $1-1.5 \mathrm{~cm}$. Echocardiographic indexes were obtained according to the recommendations of the American Society of Echocardiography (44). Rats ( $n=8$ rats per group) were anesthetized with isoflurane, and the hair on the chests of the rats was shaved. Anesthesia was maintained with the inhalant isoflurane (3-4\%, Forthane, Abbot Australasia Pty, Botany, Australia) that was delivered with oxygen $(0.5 \mathrm{l} / \mathrm{min})$ through an open-circuit inhalation anesthetic apparatus (Fluotec mark 2; Cyprane, Keighley, England). Warmed coupling gel was applied to the skin to ensure good contact with the L $12-5 \mathrm{MHz}$ transducer, and the hearts of the animals were located using B-mode imaging.

Morphological and functional analyses of the heart were performed in pulsed Doppler and M-mode, with each variable being measured over five to six consecutive traces and the mean peak amplitude of each variable being used for the analyses. The measured parameters were heart rate, LVIDd, LVIDs, PW thickness, and septal thickness. These investigations allowed the determination of left ventricle FS from the equation: $\mathrm{FS}=(($ LVIDd - LVIDs $) /$ LVIDd $) \times 100 \%(45)$.

\section{Tissue Collection and Sampling}

At $32 \mathrm{wk}$ of age, the rats were anesthetized with inactin $(150 \mathrm{mg} / \mathrm{kg})$ and then administered a lethal dose of sodium pentobarbitone $(80-100 \mathrm{mg} / \mathrm{kg}$ intraperitoneally). The abdominal cavity was exposed and a catheter was inserted into the abdominal aorta. Heparin sodium, papaverine hydrochloride, and potassium chloride were administered via the aortic catheter to prevent the blood from clotting, to maximally dilate the vasculature, and to arrest the heart in diastole, respectively (16). The animals were then perfusion-fixed in a retrograde fashion via the abdominal aorta with $4 \%$ paraformaldehyde in $0.1 \mathrm{~mol} / \mathrm{l}$ phosphate buffer.

\section{Assessment of Cardiac Fibrosis}

The hearts were excised, trimmed of fat and connective tissue, weighed, and sliced into $1.5 \mathrm{~mm}$ coronal slices. Every second slice of heart tissue (with the first slice chosen randomly) was embedded in paraffin, sectioned at $5 \mu \mathrm{m}$, and stained with $0.01 \%$ sirius red diluted in picric acid (16).

Interstitial/reparative fibrosis. A systematic uniform random sample of eight fields per section (eight fields from the LV+S and two fields from the RV) was undertaken to analyze the levels of interstitial/ reparative fibrosis (16). The collagen area was determined by measuring the area of red-stained tissue within a given field, and the relative proportion of interstitial/reparative fibrosis was subsequently quantified using image analysis software (Image-Pro Plus Version 6.0; Media Cybernetics, Bethesda, MD) as previously described (46).

Perivascular fibrosis. For the study of perivascular fibrosis, each LV+S and RV picrosirius red-stained section was initially scanned under $\times 20$ magnification, and two myocardial arteries (ranging in diameter from 150 to $400 \mu \mathrm{m}$ ) were sampled in accordance with the method described by Nyengaard and Marcussen (47). In order to determine the area of the lumen and adventitia of each artery, the outlines of the lumen and the adventitia were traced under $\times 200$ magnification, and the crosssectional area of the media of the same vessels was also measured. The areas of the lumen, media, and adventitia were subsequently calculated using image analysis software (Image-Pro Plus Version 6.0) (46).

The area of the adventitia was taken as a measure of perivascular fibrosis. To account for differences in the sizes of the arteries sampled, the adventitia:lumen ratio was calculated as a relative measure of perivascular fibrosis (46). The area of the media was measured, and the media:lumen ratio for the myocardial arteries was also determined (46).

\section{Statistical Analysis}

All data are expressed as mean \pm SEM. Data were statistically analyzed using GraphPad Prism (version 5.00; GraphPad Software, San Diego, CA). A two-way ANOVA was applied to data relating to the animals at $32 \mathrm{wk}$ of age; the factors considered were maternal diet $\left(P_{\mathrm{D}}\right.$; LPD or NPD), hyperglycemic treatment $\left(P_{\mathrm{T}}\right.$; control, mild, or moderate), and their interaction $\left(P_{\mathrm{D} \times \mathrm{T}}\right)$. Changes in blood pressure over time were analyzed using a two-way ANOVA with repeated measures. A one-way ANOVA, followed by a Tukey's post hoc test, was also applied to examine whether there were significant differences between specific LPD groups and their NPD-matched controls (LPD control vs. NPD control, mildly hyperglycemic LPD vs. mildly hyperglycemic NPD, and moderately hyperglycemic LPD vs. moderately hyperglycemic NPD) and to compare data within the LPD and NPD diet groups according to the level of hyperglycemia (mild and moderate groups vs. control, and mild vs. moderate). Statistical significance was accepted at the level of $P \leq 0.05$.

\section{STATEMENT OF FINANCIAL SUPPORT}

K.L. was the recipient of a National Health and Medical Research Council Postgraduate Award while undertaking this study.

\section{REFERENCES}

1. Hossain P, Kawar B, El Nahas M. Obesity and diabetes in the developing world-a growing challenge. N Engl J Med 2007;356:213-5.

2. Yajnik CS. Early life origins of insulin resistance and type 2 diabetes in India and other Asian countries. J Nutr 2004;134:205-10.

3. van Dieren S, Beulens JW, van der Schouw YT, Grobbee DE, Neal B. The global burden of diabetes and its complications: an emerging pandemic. Eur J Cardiovasc Prev Rehabil 2010;17:Suppl 1:S3-8.

4. Colagiuri R. Diabetes: a pandemic, a development issue or both? Expert Rev Cardiovasc Ther 2010;8:305-9.

5. Fuller JH, Elford J, Goldblatt P, Adelstein AM. Diabetes mortality: new light on an underestimated public health problem. Diabetologia 1983;24:336-41.

6. Fagot-Campagna A, Bourdel-Marchasson I, Simon D. Burden of diabetes in an aging population: prevalence, incidence, mortality, characteristics and quality of care. Diabetes Metab 2005;31 Spec No 2:5S35-52.

7. Garcia MJ, McNamara PM, Gordon T, Kannel WB. Morbidity and mortality in diabetics in the Framingham population. Sixteen year follow-up study. Diabetes 1974;23:105-11.

8. Shindler DM, Kostis JB, Yusuf S, et al. Diabetes mellitus, a predictor of morbidity and mortality in the Studies of Left Ventricular Dysfunction (SOLVD) Trials and Registry. Am J Cardiol 1996;77:1017-20.

9. Frustaci A, Kajstura J, Chimenti C, et al. Myocardial cell death in human diabetes. Circ Res 2000;87:1123-32.

10. Asbun J, Villarreal FJ. The pathogenesis of myocardial fibrosis in the setting of diabetic cardiomyopathy. J Am Coll Cardiol 2006;47:693-700.

11. Borisov AB, Ushakov AV, Zagorulko AK, et al. Intracardiac lipid accumulation, lipoatrophy of muscle cells and expansion of myocardial infarction in type 2 diabetic patients. Micron 2008;39:944-51.

12. Kannel WB, Hjortland M, Castelli WP. Role of diabetes in congestive heart failure: the Framingham study. Am J Cardiol 1974;34:29-34.

13. Kaijser M, Bonamy AK, Akre O, et al. Perinatal risk factors for ischemic heart disease: disentangling the roles of birth weight and preterm birth. Circulation 2008;117:405-10.

14. Barker DJ, Winter PD, Osmond C, Margetts B, Simmonds SJ. Weight in infancy and death from ischaemic heart disease. Lancet 1989;2:577-80. 
15. Zhang L. Prenatal hypoxia and cardiac programming. J Soc Gynecol Investig 2005;12:2-13.

16. Lim K, Zimanyi MA, Black MJ. Effect of maternal protein restriction in rats on cardiac fibrosis and capillarization in adulthood. Pediatr Res 2006;60:83-7.

17. Cheema KK, Dent MR, Saini HK, Aroutiounova N, Tappia PS. Prenatal exposure to maternal undernutrition induces adult cardiac dysfunction. Br J Nutr 2005;93:471-7.

18. Camm EJ, Hansell JA, Kane AD, et al. Partial contributions of developmental hypoxia and undernutrition to prenatal alterations in somatic growth and cardiovascular structure and function. Am J Obstet Gynecol 2010;203:495.e24-34.

19. Battista MC, Calvo E, Chorvatova A, Comte B, Corbeil J, Brochu M. Intra-uterine growth restriction and the programming of left ventricular remodelling in female rats. J Physiol (Lond) 2005;565(Pt 1):197-205.

20. Rueda-Clausen CF, Morton JS, Davidge ST. Effects of hypoxia-induced intrauterine growth restriction on cardiopulmonary structure and function during adulthood. Cardiovasc Res 2009;81:713-22.

21. Zimanyi MA, Denton KM, Forbes JM, et al. A developmental nephron deficit in rats is associated with increased susceptibility to a secondary renal injury due to advanced glycation end-products. Diabetologia 2006;49:801-10.

22. Zimanyi MA, Bertram JF, Black MJ. Does a nephron deficit in rats predispose to salt-sensitive hypertension? Kidney Blood Press Res 2004; 27:239-47.

23. Swali A, McMullen S, Langley-Evans SC. Prenatal protein restriction leads to a disparity between aortic and peripheral blood pressure in Wistar male offspring. J Physiol (Lond) 2010;588(Pt 19):3809-18.

24. Brennan KA, Kaufman S, Reynolds SW, et al. Differential effects of maternal nutrient restriction through pregnancy on kidney development and later blood pressure control in the resulting offspring. Am J Physiol Regul Integr Comp Physiol 2008;295:R197-205.

25. Hoppe CC, Evans RG, Moritz KM, et al. Combined prenatal and postnatal protein restriction influences adult kidney structure, function, and arterial pressure. Am J Physiol Regul Integr Comp Physiol 2007;292:R462-9.

26. Tonkiss J, Trzcinska M, Galler JR, Ruiz-Opazo N, Herrera VL. Prenatal malnutrition-induced changes in blood pressure: dissociation of stress and nonstress responses using radiotelemetry. Hypertension 1998;32:108-14.

27. Briscoe TA, Rehn AE, Dieni S, et al. Cardiovascular and renal disease in the adolescent guinea pig after chronic placental insufficiency. Am J Obstet Gynecol 2004;191:847-55.

28. Veille JC, Hanson R, Sivakoff M, Hoen H, Ben-Ami M. Fetal cardiac size in normal, intrauterine growth retarded, and diabetic pregnancies. Am J Perinatol 1993;10:275-9.

29. Tsyvian P, Malkin K, Artemieva O, Blyakhman F, Wladimiroff JW. Cardiac ventricular performance in the appropriate- for-gestational age and smallfor-gestational age fetus: relation to regional cardiac non-uniformity and peripheral resistance. Ultrasound Obstet Gynecol 2002;20:35-41.
30. de Souza Vilarinho KA, Petrucci O, Baker RS, et al. Early changes in contractility indices and fibrosis in two minimally invasive congestive heart failure models. Eur J Cardiothorac Surg 2010;37:368-75.

31. Liu SK, Magid NR, Fox PR, Goldfine SM, Borer JS. Fibrosis, myocyte degeneration and heart failure in chronic experimental aortic regurgitation. Cardiology 1998;90:101-9.

32. Kruyt ND, Biessels GJ, Devries JH, Roos YB. Hyperglycemia in acute ischemic stroke: pathophysiology and clinical management. Nat Rev Neurol 2010;6:145-55.

33. Fuller JH, Stevens LK, Wang SL. Risk factors for cardiovascular mortality and morbidity: the WHO Mutinational Study of Vascular Disease in Diabetes. Diabetologia 2001;44:Suppl 2:S54-64.

34. Wachter R, Lüers C, Kleta S, et al. Impact of diabetes on left ventricular diastolic function in patients with arterial hypertension. Eur J Heart Fail 2007;9:469-76.

35. Shehadeh A, Regan TJ. Cardiac consequences of diabetes mellitus. Clin Cardiol 1995;18:301-5.

36. Marwick TH. Diabetic heart disease. Postgrad Med J 2008;84:188-92.

37. Smit AJ, Hartog JW, Voors AA, van Veldhuisen DJ. Advanced glycation endproducts in chronic heart failure. Ann N Y Acad Sci 2008;1126:225-30.

38. De Groote P, Lamblin N, Mouquet F, et al. Impact of diabetes mellitus on long-term survival in patients with congestive heart failure. Eur Heart J 2004;25:656-62.

39. Federmann M, Hess OM. Differentiation between systolic and diastolic dysfunction. Eur Heart J 1994;15:Suppl D:2-6.

40. Braun B, Zimmermann MB, Kretchmer N, Spargo RM, Smith RM, Gracey M. Risk factors for diabetes and cardiovascular disease in young Australian aborigines. A 5-year follow-up study. Diabetes Care 1996;19:472-9.

41. Barker DJ. The intrauterine environment and adult cardiovascular disease. Ciba Found Symp 1991;156:3-10; discussion 10-6.

42. Barker DJ, Osmond C, Golding J, Kuh D, Wadsworth ME. Growth in utero, blood pressure in childhood and adult life, and mortality from cardiovascular disease. BMJ 1989;298:564-7.

43. Li F, Wang X, Capasso JM, Gerdes AM. Rapid transition of cardiac myocytes from hyperplasia to hypertrophy during postnatal development. J Mol Cell Cardiol 1996;28:1737-46.

44. Nagueh SF, Appleton CP, Gillebert TC, et al. Recommendations for the evaluation of left ventricular diastolic function by echocardiography. Eur J Echocardiogr 2009;10:165-93.

45. Brown L, Fenning A, Chan V, et al. Echocardiographic assessment of cardiac structure and function in rats. Heart Lung Circ 2002;11:167-73.

46. Bensley JG, Stacy VK, De Matteo R, Harding R, Black MJ. Cardiac remodelling as a result of pre-term birth: implications for future cardiovascular disease. Eur Heart J 2010;31:2058-66.

47. Nyengaard JR, Marcussen N. The number of glomerular capillaries estimated by an unbiased and efficient stereological method. J Microsc 1993;171(Pt 1):27-37. 